\title{
The Application of Classification and Regression Trees for the Triage of Women for Referral to Colposcopy and the Estimation of Risk for Cervical Intraepithelial Neoplasia: A Study Based on 1625 Cases with Incomplete Data from Molecular Tests
}

\author{
Abraham Pouliakis, ${ }^{1}$ Efrossyni Karakitsou, ${ }^{2}$ Charalampos Chrelias, ${ }^{3}$ \\ Asimakis Pappas, ${ }^{3}$ Ioannis Panayiotides, ${ }^{4}$ George Valasoulis, ${ }^{5,6}$ Maria Kyrgiou, ${ }^{7,8}$ \\ Evangelos Paraskevaidis, ${ }^{5}$ and Petros Karakitsos ${ }^{1}$ \\ ${ }^{1}$ Department of Cytopathology, University of Athens Medical School, Attikon University Hospital, \\ Rimini 1, Haidari, 12462 Athens, Greece \\ ${ }^{2}$ Biomedical Engineering Laboratory, National Technical University of Athens, Iroon Politechniou 9, Zografou, \\ 15780 Athens, Greece \\ ${ }^{3} 3$ rd Department of Obstetrics and Gynecology, University of Athens Medical School, Attikon University Hospital, \\ Rimini 1, Haidari, 12462 Athens, Greece \\ ${ }^{4}$ 2nd Department of Pathology, University of Athens Medical School, Attikon University Hospital, \\ Rimini 1, Haidari, 12462 Athens, Greece \\ ${ }^{5}$ Department of Obstetrics \& Gynecology, University Hospital of Ioannina, Stavros Niarchos Avenue, 45500 Ioannina, Greece \\ ${ }^{6}$ Department of Obstetrics \& Gynaecology, Worthing Hospital, Western Sussex Hospitals NHS Foundation Trust, \\ Worthing BN11 2DH, UK \\ ${ }^{7}$ Department of Surgery and Cancer, Institute of Reproductive \& Developmental Biology, Faculty of Medicine, \\ Imperial College, London W12 0NN, UK \\ ${ }^{8}$ West London Gynaecological Cancer Center, Queen Charlotte's and Chelsea-Hammersmith Hospital, \\ Imperial Healthcare NHS Trust, London W12 0HS, UK
}

Correspondence should be addressed to Abraham Pouliakis; apou1967@gmail.com

Received 24 October 2014; Accepted 14 February 2015

Academic Editor: Ondrej Topolcan

Copyright (C) 2015 Abraham Pouliakis et al. This is an open access article distributed under the Creative Commons Attribution License, which permits unrestricted use, distribution, and reproduction in any medium, provided the original work is properly cited.

Objective. Nowadays numerous ancillary techniques detecting HPV DNA and mRNA compete with cytology; however no perfect test exists; in this study we evaluated classification and regression trees (CARTs) for the production of triage rules and estimate the risk for cervical intraepithelial neoplasia (CIN) in cases with ASCUS+ in cytology. Study Design. We used 1625 cases. In contrast to other approaches we used missing data to increase the data volume, obtain more accurate results, and simulate real conditions in the everyday practice of gynecologic clinics and laboratories. The proposed CART was based on the cytological result, HPV DNA typing, HPV mRNA detection based on NASBA and flow cytometry, p16 immunocytochemical expression, and finally age and parous status. Results. Algorithms useful for the triage of women were produced; gynecologists could apply these in conjunction with available examination results and conclude to an estimation of the risk for a woman to harbor CIN expressed as a probability. Conclusions. The most important test was the cytological examination; however the CART handled cases with inadequate cytological outcome and increased the diagnostic accuracy by exploiting the results of ancillary techniques even if there were inadequate missing data. The CART performance was better than any other single test involved in this study. 


\section{Introduction}

Cervical cancer (CC) is the third most common cancer and the fourth leading cause of cancer death in females worldwide [1]. More than $85 \%$ of these cases and deaths are in developing countries; this is due to lack of screening that may allow detection of precancerous and early stage cervical cancer. Despite the advances in screening, cervical cancer remains a serious problem of public health even in developed countries, due to the high percentage of detection failures [2].

$\mathrm{CC}$ is known to be caused almost always by human papillomavirus (HPV) infection which is the commonest sexually transmitted infection worldwide. About 100 types of HPV virus that can infect humans have been identified. Among them, 15 are oncogenic and can cause CC. Improved understanding of HPV infection and the natural history of cervical neoplasia have resulted in the addition of the HPV DNA test along with the Pap test and frequently a competing test.

Nowadays, ancillary techniques for CC screening are available. These include HPV DNA typing and mRNA identification of the viral E6/E7 oncogenes that are linked to oncogenic activation. Among them, mRNA typing with nucleic acid based amplification (NASBA) [3] and mRNA-FlowFISH techniques in screening programs produced promising results in increasing PPV and reducing unnecessary recalls and referrals to colposcopy [4-7]. At the same time, it was reported that the immunocytochemical detection of p16 can increase the diagnostic accuracy of the Pap test [8].

Several published studies in the literature attempted to clarify the role of each technique as a unique test to substitute the Pap test [3-7, 9-17]. By the detailed analysis of these, it can be concluded that the performance measures of the methods under control differ significantly, affected by the disease incidence and the prevalence of HPV infection in the population study group; thus, application of a single method, even if it offers a level of protection, does not determine reliably the risk for individual women to harbor cervical intraepithelial neoplasia (CIN). However, from the metaanalysis of published studies [9-12] it is evident that the sensitivity of Pap test combined with the HPV DNA test is higher than the sensitivity of each individual method.

Computer science and artificial intelligence enabled the development of computer assisted systems for the support of clinical diagnosis or therapeutic and treatment decisions. Various classification techniques such as neural networks [18-29], discriminant analysis [18, 30-32], classification and regression trees (CARTs) [33-35], or genetic algorithms [36] have been used in medicine. The application of new molecular techniques that are nowadays used in the diagnostic cytology laboratory [37] improves the accuracy of the final diagnosis in comparison to that of cytology alone.

Among the various decision support techniques (CARTs) is an attractive statistical approach to extract knowledge from data as they are straightforward to construct and easily understandable by physicians. The application of these systems produces simple decision algorithms linked with probabilities that can be promising to define triage rules and perhaps give a better understanding of the disease.
The aim of this study was to investigate the potential role of CARTs applied on various diagnostic variables measured in the modern cytopathology laboratory and to build algorithms for the triage of individual cases. Special focus was given to design the study as pragmatic as possible: thus, (1) cases from two different parts of the country were selected, (2) a part of the cases were considered to be negative as no histological confirmation could be obtained due to ethical reasons, and (3) inadequate test results (i.e., missing data) were included as this is the cytopathology laboratory reality.

\section{Materials and Methods}

2.1. Involved Institutes and Ethics. Our study involved the 3rd Department of Obstetrics and Gynecology, the Department of Cytopathology and the 2nd Department of Pathology, all three hosted in "Attikon" University Hospital, Medical School of Athens University, and the Department of Obstetrics and Gynecology of University Hospital of Ioannina City. The study was approved by the University Hospital Ethics Boards and participating women signed an informed consent (ICON) form to allow use of their epidemiologic, diagnostic, and ancillary test data.

2.2. Cytology. All cytological and ancillary examinations were based on ThinPrep liquid based cytology (LBC) material obtained before colposcopical examination. The smears were routinely prepared for cytological examination and the remaining material in the ThinPrep vial was used for additional evaluation of biomarkers related to the HPV lifecycle. The smears were assessed by experienced cytopathologists. Histological material was obtained during colposcopy and/or during treatment by conization. The obtained histological samples were fixed and prepared according to standard histopathology protocols.

The cytological findings for each woman were formulated according to the revised Bethesda classification system (TBS2001 system) [38, 39].

2.3. Histological Confirmation. The histological diagnosis was the golden standard and was used as the target category of each woman. Punch biopsies were performed by experienced colposcopists (in practice for more than 10 years) as part of the study protocol. The three-tiered cervical intraepithelial neoplasia grading system was used for reporting histological diagnosis. Clinically negative $(\mathrm{CN})$ cases were included in the study. These were defined as $\mathrm{CN}$ if the cytology, colposcopy, and the CLART Human Papillomavirus 2 HPV DNA test (see Section 2.4) were all negative. Despite the lack of histological biopsies due to ethical hurdles, these women were included and analyzed in a target category of less than CIN2. The correlation of the cytological results in relation to histology is presented in Table 1.

2.4. Molecular Tests. In relation to the HPV lifecycle biomarkers we used (a) HPV DNA typing using the CLART Human Papillomavirus 2 (GENOMICA) kit for the simultaneous detection of 35 different HPV genotypes by PCR 
TABLE 1: Correlation of the cytological with the histological diagnosis.

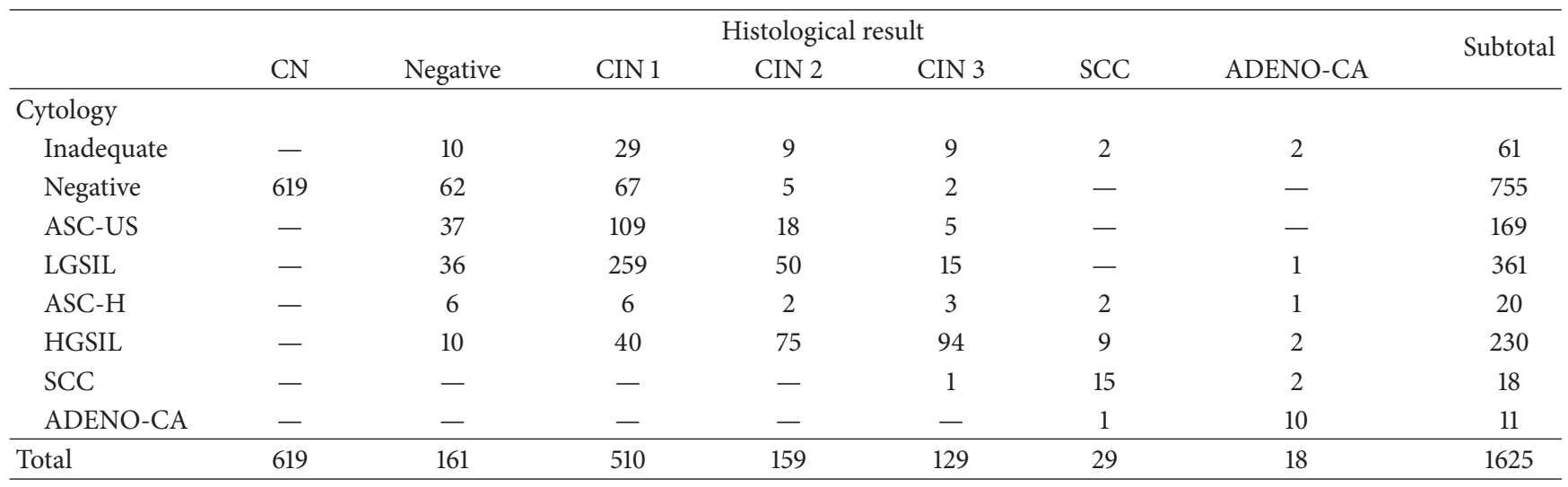

amplification of a fragment within the highly conserved L1 region of the virus [40]; (b) NASBA assays [41] (NucliSENS EasyQ HPV v1.0) that were used for the identification of E6/E7 mRNA of the HPV types: 16, 18, 31, 33, and 45; (c) the PermiFlow (Invirion Diagnostics, LLC, Oak Brook, IL) kit for the identification of E6/E7 mRNA expression of high risk HPV using flow cytometry [6]; and (d) the immunocytochemical expression of p16 using the CINtec Cytology Kit [42]. In addition to pure medical data, epidemiologic features were involved as well, specifically woman age and parous status.

Within the clinical laboratory, it is not infrequent that an ancillary test produces invalid results or the biological material that remains in the vial is not adequate to perform additional tests; therefore, it is not guaranteed that there are available sets of such data for all women participating in the study. Additionally parous details were not available for all women as such data often were not considered important and referral forms were incomplete.

2.5. Golden Standard. Our target was to classify each woman into one of the following categories: (a) <CIN2, which included the histologically negative and CIN-1 cases as well as the CN cases, and (b) $\geq \mathrm{CIN} 2$, which included the histological categories: CIN-2, CIN-3, SCC, and ADENO-CA.

2.6. Data Formulation. For each case, a vector of 50 variables was created (Table 2); this had the result of the cytological examination expressed according to the Bethesda system. Results of the HPV DNA test examination were expressed as 35 individual values (either positive or negative), one for each HPV DNA genotype; additionally, in relation to the found subtypes five other variables were investigated: the existence of high risk, low risk, or any type as well as the number of high risk and the number of low risk types that were identified. For the NASBA HPV mRNA typing, we used the result for each individual HPV type $(16,18,31,33$, and 45$)$. The result of the PermiFlow test was involved using two methods, either as a percentage or as positive or negative (the cut-off value to assign a flow cytometry result was 1.5\%); in addition, the result of the immunocytochemical expression of p16 was included. Finally, two other variables were entered to the tree construction process: woman age and parous status. For all variables, if there were no data, the value was left blank or declared inadequate indicating that there was no valid result or there was not adequate material in the ThinPrep vial to perform additional examinations.

2.7. Statistical Techniques and Modeling. The CART model was created using IBM SPSS Statistics 19 for Windows (SPSS Inc., Chicago, USA). The CART algorithm is possible to be configured and use a specific feature at the first node of the tree; however, in this study CART was allowed to select as first test the test with the highest overall accuracy. To assess the performance, various statistical measures were extracted: specificity, sensitivity, positive and negative predictive value (PPV and NPV), false positive and false negative rates (FPR and NPR), and overall accuracy (OA).

\section{Results}

In total 1006 histologically confirmed cases (161 without evidence of CIN or malignancy, 510 CIN-1, 159 CIN-2, $129 \mathrm{CIN}-3$, and 47 cervical cancer cases (29 squamous cell carcinomas (SCC) and 18 adenocarcinomas (ADENO-CA))) were included in this study and additionally $619 \mathrm{CN}$ cases. The correlation of the cytological versus the histological outcome of our material appears in Table 1. In our material, the percentage of valid data (i.e., after excluding inadequate, invalid, and unsatisfactory results) was for the cytological examination $96.25 \%$, for ARRAYS 91.94\%, for NASBA $67.75 \%$, for flow cytometry $81.54 \%$, and for p16 68.68\%.

For the construction of the CART model, the CHAID algorithm was used; the CART architecture was 20-5-10; that is, each parent node was forced to 20 or more vectors, and each terminal node had more than 5 vectors, and the tree depth was not allowed to grow more than 10 levels. The system was pruned to obtain simpler forms, the significance level for splitting a node was set to 0.05 , the chi-square statistic was based on the likelihood ratio and the significance values were adjusted using the Bonferroni method, resplitting of merged categories within one node was allowed, the age and flow 
TABLE 2: Variables entered to the model.

\begin{tabular}{|c|c|c|}
\hline Variable name & Description & Value range \\
\hline Cytology & $\begin{array}{l}\text { The result of the cytological } \\
\text { examination expressed according to } \\
\text { the Bethesda } 2009 \text { system }\end{array}$ & $\begin{array}{l}\text { WNL, ASC-US, LGSIL, ASC-H, HGSIL, SCC, } \\
\text { ADENO CA, and < blank > if there is no result }\end{array}$ \\
\hline $\begin{array}{l}\text { HPV DNA arrays } \\
\text { A6, A11, A16, A18, A26, A31, A33, A35, A39, } \\
\text { A40, A42, A43, A44, A45, A51, A52, A53, A54, } \\
\text { A56, A58, A59, A61, A62, A66, A68, A70, A71, } \\
\text { A72, A73, A81, A82, A83, A84, A85, and A89 }\end{array}$ & $\begin{array}{l}\text { The existence of individual subtypes } \\
\text { according to the arrays examination }\end{array}$ & $\begin{array}{l}0 \text { if the specific subtype is not found } \\
1 \text { if the specific subtype is found } \\
<\text { blank }>\text { if there is no result }\end{array}$ \\
\hline Has HR & $\begin{array}{l}\text { Positive if one or more high risk } \\
\text { subtypes were found during typing }\end{array}$ & Positive, negative, or missing \\
\hline Has LR & $\begin{array}{l}\text { Positive if one or more low risk } \\
\text { subtypes were found during typing }\end{array}$ & Positive, negative, or missing \\
\hline Has any type & $\begin{array}{l}\text { Positive if one or more subtypes were } \\
\text { found during typing }\end{array}$ & Positive, negative, or missing \\
\hline No HR & $\begin{array}{l}\text { The number of high risk subtypes that } \\
\text { were found during typing }\end{array}$ & An integer or missing \\
\hline No LR & $\begin{array}{l}\text { The number of low risk subtypes that } \\
\text { were found during typing }\end{array}$ & An integer or missing \\
\hline N16, N18, N31, N33, and N45 & $\begin{array}{l}\text { The result of the E6/E7 mRNA test for } \\
\text { the specific HPV subtype }\end{array}$ & $\begin{array}{l}0 \text { if negative } \\
1 \text { if positive } \\
<\text { blank }>\text { if there is no result }\end{array}$ \\
\hline Flow & $\begin{array}{l}\text { The result of the identification of } \\
\text { E6/E7 mRNA expression of high risk } \\
\text { HPV using flow cytometry technique }\end{array}$ & $\begin{array}{l}0 \text { if negative }(<1.5 \%) \\
1 \text { if positive }(>1.5 \%) \\
<\text { blank }>\text { if there is no result }\end{array}$ \\
\hline Flow \% & $\begin{array}{l}\text { The result of E6/E7 mRNA expression } \\
\text { of high risk HPV using flow cytometry } \\
\text { technique expressed as a percentage }\end{array}$ & A number or $<$ blank $>$ if there is no result \\
\hline p16 & $\begin{array}{l}\text { The result of the p16 } \\
\text { immunocytochemical examination }\end{array}$ & $\begin{array}{l}0 \text { if negative } \\
1 \text { if positive } \\
<\text { blank }>\text { if there is no result }\end{array}$ \\
\hline Age & $\begin{array}{l}\text { The woman age at the time of } \\
\text { examination }\end{array}$ & A positive number \\
\hline Parous & Woman parous status & $\begin{array}{l}1 \text { if she has born one or more children and } 0 \text { if } \\
\text { not }\end{array}$ \\
\hline
\end{tabular}

percentage intervals were set to 10 (i.e., flow and age values had 10 levels), and finally the maximum number of iterations was 500.

The CART architecture appears in Figure 1. It is worth noting that the role of the cytological examination result is dominant and that characteristics, such as the woman's age and the majority of HPV subtypes as were identified by the HPV subtyping test, were not found significant to be included. An example of a method of tree usage in practice is as follows.

(i) The user starts from the top node and examines the value of the proposed characteristic, in our case the cytological examination outcome.

(ii) According to the value the user navigates to the appropriate node; in our case if the cytological examination is inadequate, we examine the flow result and a negative result leads the user to a probability for the case to be benign equal to $93.9 \%$ while if there is no result or the outcome of flow cytometry is positive then the user may examine the existence on HPV subtype 16 within the mRNA test, and interestingly a negative result provides a probability for malignancy equal to $93.8 \%$ as 15 out of the 16 cases with this profile were $\geq$ CIN2 in histology.

(iii) During navigation to the tree, the user may choose to stay in the proposed node if he/she is satisfied by the risk danger (probability) otherwise may examine the next proposed feature to find a more accurate result.

(iv) The steps are repeated down to the terminal nodes if the user is not satisfied from the proposed risk levels from the previous parent nodes.

In a second example, the user may start with a negative cytological examination result; according to the lab performance, the probability for such a case to be positive is very small $(0.9 \%)$. However, if more guarantees are required for this result, an HPV DNA test may be performed, a positive outcome on HPV subtype 16 reduces the probability of a case to be less than CIN-2 from $99.1 \%$ to $66.7 \%$ (4 out 


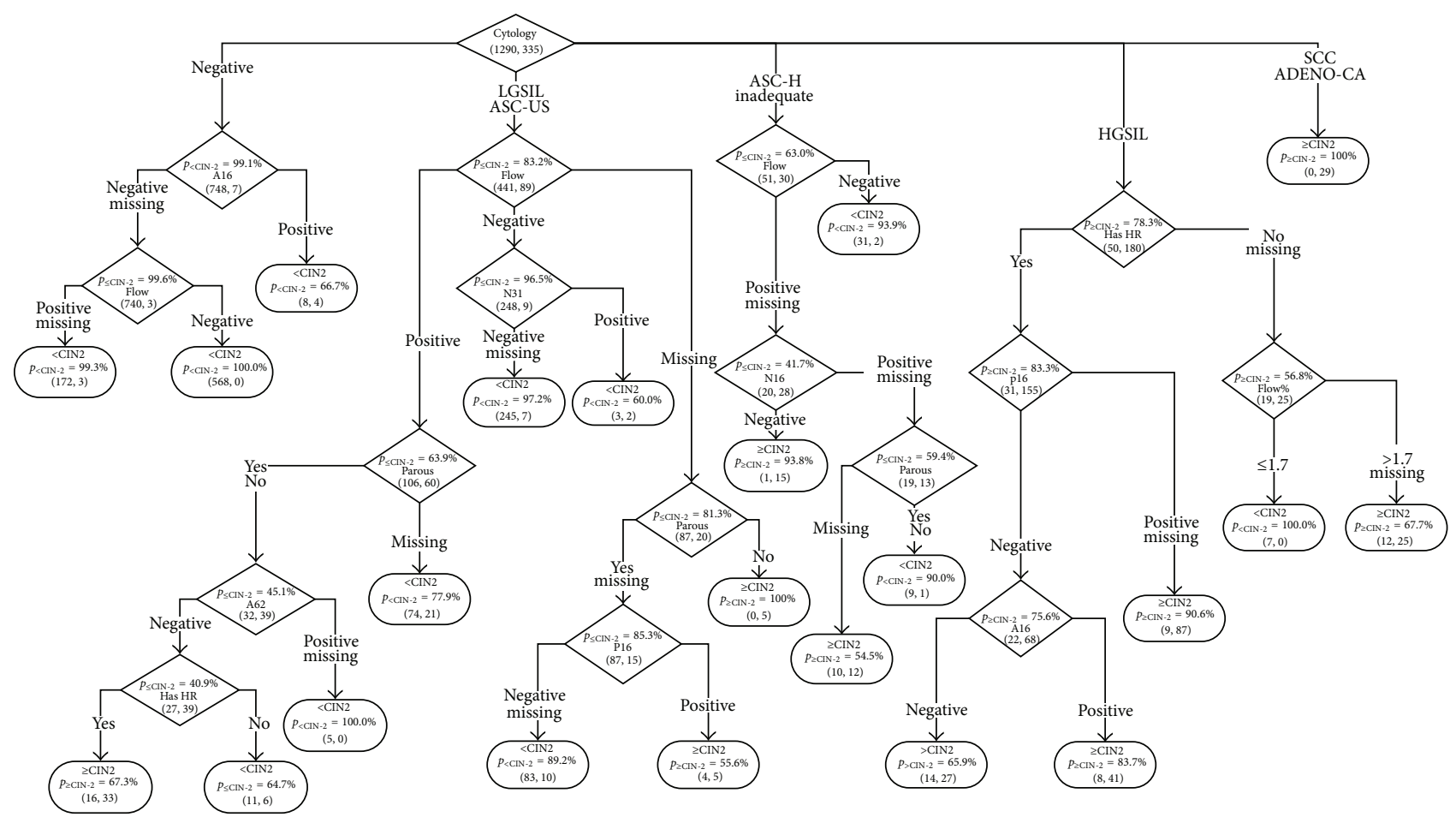

FIGURE 1: Structure of the CART model.

of the 12 cases with negative cytology and positive HPV DNA subtype 16 were finally found histologically CIN-2 or worst), and a negative or invalid result on test for HPV subtype 16 combined with a negative result for flow cytometry practically assures the woman that the probability to have a lesion worse than CIN-2 is negligible, as 568 cases with this profile in our material had less than CIN-2 and no case was found with equal or more than CIN-2 lesion.

A third example is related to the cases that are ASCUS or LGSIL in cytology; if the triage is based only on the cytological examination, all these women should be referred to colposcopy. However, in our material 441 cases were $<$ CIN2 and 89 were $\geq$ CIN2 (in total 530 women); thus, the probability to have $<\mathrm{CIN} 2$ is $83.2 \%$, and if additional material is available in the vial, then a flow examination may be more helpful for the triage; in particular, a negative result in the flow examination gives a probability for $<$ CIN2 equal to $96.5 \%$, while a positive result indicates that this woman has more chances to harbor $\geq \operatorname{CIN} 2$ (46.1\% Figure 1$)$, and in such case these women could be immediately referred to colposcopy. This approach would allow reduction of referrals to colposcopy by 257, in more detail from 530 to 273; therefore, about half of the women $(51.51 \%)$ could avoid immediate referral to colposcopy.

The assignment matrix of CART results appears in Table 3; actually the system classified 1216 out of the 1290 $<$ CIN2 cases and 279 out of the $335 \geq$ CIN2 cases, and the statistics show that the model had sensitivity: $83.28 \%$, specificity: $94.26 \%$, PPV: $79.04 \%$, NPV: $95.60 \%$, FPR: $5.74 \%$, FNR: $16.72 \%$, and OA: $92.00 \%$. In order to assess the CART
TABLE 3: CART results.

\begin{tabular}{lccc}
\hline \multirow{2}{*}{ Actual category } & \multicolumn{2}{c}{ Predicted category } & Grand total \\
& $<$ CIN 2 & $\geq$ CIN 2 & \\
\hline$<$ CIN 2 & 1216 & 74 & $\mathbf{1 2 9 0}$ \\
$\geq$ CIN 2 & 56 & 279 & $\mathbf{3 3 5}$ \\
\hline Grand total & $\mathbf{1 2 7 2}$ & $\mathbf{3 5 3}$ & $\mathbf{1 6 2 5}$ \\
\hline
\end{tabular}

performance in relation to the performance of each individual examination, the related statistics were extracted.

Specifically in Table 4 are summarized the performance metrics of the CART model, of the cytological examination using the ASC-US cases as a cutoff (i.e., all cases that were ASC-US and above in cytology were considered positive and referred to colposcopy) and similarly using an ASC-H cytological outcome as cutoff. For the HPV DNA typing, three alternative methods were evaluated: a woman was considered to be at risk and referred to colposcopy if (1) any HPV type was found, (2) only if a high risk type was found, and (3) only if 16 or 18 subtypes were identified. Finally, in Table 4 are presented the performance metrics for mRNA test using NASBA or flow cytometry and p16; that is, a case was considered to be at risk if any subtype was found in the NASBA examination or if a flow cytometry result was positive or screening of a p16 slide gave a positive result.

Finally in order to allow a more detailed evaluation of the methodology, in Table 5 are summarized the results of the histological outcome (blocks of rows) along with the 
TABLE 4: Performance of CART and individual examinations.

\begin{tabular}{|c|c|c|c|c|c|c|c|c|c|}
\hline & CART & $\begin{array}{l}\text { Cytology } \\
\text { using } \\
\text { cutoff } \\
\text { ASC-US+ }\end{array}$ & $\begin{array}{l}\text { Cytology } \\
\text { using } \\
\text { cutoff } \\
\text { ASC-H+ }\end{array}$ & $\begin{array}{c}\text { Arrays } \\
\text { (positive if } \\
\text { any type } \\
\text { was found) }\end{array}$ & $\begin{array}{c}\text { Arrays } \\
\text { (positive if a } \\
\text { high risk type } \\
\text { was found) }\end{array}$ & $\begin{array}{c}\text { Arrays } \\
\text { (positive if } \\
\text { subtype } 16 \text { or } \\
18 \text { was found) }\end{array}$ & $\begin{array}{l}\text { NASBA } \\
\text { (positive if } \\
\text { any type } \\
\text { was found) }\end{array}$ & $\begin{array}{l}\text { Flow cytometry } \\
\text { (positive if the } \\
\text { result is positive } \\
>1.5 \% \text { ) }\end{array}$ & p16 \\
\hline Sensitivity & $83.28 \%$ & $97.76 \%$ & $69.33 \%$ & $87.71 \%$ & $84.72 \%$ & $52.82 \%$ & $69.65 \%$ & $88.19 \%$ & $57.21 \%$ \\
\hline Specificity & $94.26 \%$ & $59.79 \%$ & $95.04 \%$ & $70.49 \%$ & $75.02 \%$ & $90.28 \%$ & $87.09 \%$ & $79.60 \%$ & $93.57 \%$ \\
\hline PPV & $79.04 \%$ & $37.82 \%$ & $77.78 \%$ & $42.86 \%$ & $46.11 \%$ & $57.82 \%$ & $62.15 \%$ & $48.49 \%$ & $69.68 \%$ \\
\hline NPV & $95.60 \%$ & $99.07 \%$ & $92.53 \%$ & $95.79 \%$ & $95.11 \%$ & $88.35 \%$ & $90.41 \%$ & $96.87 \%$ & $89.44 \%$ \\
\hline FPR & $5.74 \%$ & $40.21 \%$ & $4.96 \%$ & $29.51 \%$ & $24.98 \%$ & $9.72 \%$ & $12.91 \%$ & $20.40 \%$ & $6.43 \%$ \\
\hline FNR & $16.72 \%$ & $2.24 \%$ & $30.67 \%$ & $12.29 \%$ & $15.28 \%$ & $47.18 \%$ & $30.35 \%$ & $11.81 \%$ & $42.79 \%$ \\
\hline $\mathrm{OA}$ & $92.00 \%$ & $67.39 \%$ & $89.90 \%$ & $73.96 \%$ & $76.97 \%$ & $82.73 \%$ & $83.02 \%$ & $81.13 \%$ & $86.11 \%$ \\
\hline Valid results & 1625 & 1564 & 1564 & 1494 & 1494 & 1494 & 1101 & 1325 & 1116 \\
\hline $\begin{array}{l}\% \text { of valid } \\
\text { results }\end{array}$ & $100.00 \%$ & $96.25 \%$ & $96.25 \%$ & $91.94 \%$ & $91.94 \%$ & $91.94 \%$ & $67.75 \%$ & $81.54 \%$ & $68.68 \%$ \\
\hline
\end{tabular}

cytological categories to which the cases were assigned (rows) in combination with the CART model outcome (columns).

\section{Discussion and Conclusions}

Test Papanicolaou is viewed as the most successful CC test [43] if it is repeatedly applied. However, CC is not yet eliminated even in countries with well-organized cervical cancer screening programs. There are many available options for the application of biomarkers in the triage of abnormal cases [17, 44-51]; however, these are either highly sensitive or highly specific, but not both at the same time. Nowadays, there is no consensus for the optimal management of women with abnormal Pap smears and equally not infrequently women with negative cytology are found to have a high-grade lesion $\geq$ CIN2 histologically. Women with an ASC-US result in cytology present more complex management problems. The widely accepted management options of such cases are either immediate referral to colposcopy or surveillance with repeated Pap tests. The first option can overload colposcopy clinics and may lead to overintervention and overtreatment due to subtle findings. Overtreatment commonly has negative psychological effects with increasing anxiety and may further increase the risk for long-term perinatal morbidity in subsequent pregnancies [52]. Conversely, repeat cytology with surveillance has an inherent risk of missing HGSILs, dependent on the laboratory performance, has the risk of poor compliance, and may inversely increase the psychological burden for women with cytological abnormalities that are not further assessed. It is clear that we need more accurate diagnostic tools in order to limit the number of unnecessary colposcopic referrals without compromising the detection of high-grade disease.

In our material the percentage of $\geq \mathrm{CIN} 2$ cases in the total of the cases given as ASC-US was $23 / 169=13.61 \%$. Furthermore, the percentage of $\geq \mathrm{CIN} 2$ cases in the total of LgSIL cases was $66 / 361=18.28 \%$. Both percentages are in agreement with those reported by other researchers [53] that range between $5-17 \%$ and $9-16 \%$, respectively, in the published literature. On the other hand, the percentage of cases given in cytology as HGSIL and found histologically lower than CIN2 was $50 / 230=21.74 \%$. This is also consistent with the rates published in the literature $[54,55]$, demonstrating an agreement across various study settings.

Exploitation of ancillary test data for improvement of cervical intraepithelial lesions is nowadays a hot research topic with important applications. Since 2010, the Hellenic Cervical Pathology (HeCPA) Study Group is working on innovative approaches that use advanced mathematical and computing tools for the exploitation of ancillary tests that are nowadays available. Up to now, preliminary results are presented in the literature [35, 56]. In our previously published study [35], we applied CART models based on a smaller dataset, using cases that had valid examination results for all the available ancillary tests. This approach had clearly the disadvantage of a reduced usable data volume and does not capture the real life situation, that is, missing values. In addition, parameters related to woman history and demographic data were not included and the probabilities for a woman to harbor CIN were not calculated for each tree part. In two other published reports $[56,57]$ by the same group, there were applied neural networks to solve the same problem; the disadvantage of these approaches was again the requirement to have complete data for each series and no risk estimation was performed. In this study, we exploited the CART ability to handle cases with missing data and therefore increase the power of the study. The probability for each individual part of the tree was extracted. We used additional information related to women and concluded that parous is an important factor. We also extracted knowledge from our dataset in the form of triage algorithms that not only could be useful to the decisionmakers towards their requests for ancillary tests but also promote a scoring system classifying individual women as high, low, or middle risk.

According to our results, despite the multitude of features entered into the CART model (Table 2), the training algorithm identified as useful only a small number of those parameters and was finally included in the CART model (Figure 1). The major discriminating characteristic was the cytological diagnosis; in relation to typing, only the existence 
TABLE 5: CART results in relation to the cytological outcome and the histological result.

\begin{tabular}{|c|c|c|c|c|c|}
\hline \multirow{2}{*}{ Histology } & \multirow{2}{*}{ Cytology } & \multicolumn{3}{|c|}{ CART result } & \multirow{2}{*}{ Grand total } \\
\hline & & Correct & False negative & False positive & \\
\hline $\mathrm{CN}$ & Negative & 619 & & & 619 \\
\hline (CN) Total & & 619 & & & 619 \\
\hline \multirow{6}{*}{ Negative } & Inadequate & 10 & & & 10 \\
\hline & Negative & 62 & & & 62 \\
\hline & ASC-US & 36 & & 1 & 37 \\
\hline & LGSIL & 36 & & & 36 \\
\hline & ASC-H & 3 & & 3 & 6 \\
\hline & HGSIL & 2 & & 8 & 10 \\
\hline Negative total & & 149 & & 12 & 161 \\
\hline \multirow{6}{*}{ CIN 1} & Inadequate & 25 & & 4 & 29 \\
\hline & Negative & 67 & & & 67 \\
\hline & ASC-US & 107 & & 2 & 109 \\
\hline & LGSIL & 242 & & 17 & 259 \\
\hline & ASC-H & 2 & & 4 & 6 \\
\hline & HGSIL & 5 & & 35 & 40 \\
\hline CIN 1 total & & 448 & & 62 & 510 \\
\hline \multirow{6}{*}{ CIN 2} & Inadequate & 8 & 1 & & 9 \\
\hline & Negative & & 5 & & 5 \\
\hline & ASC-US & 7 & 11 & & 18 \\
\hline & LGSIL & 26 & 24 & & 50 \\
\hline & ASC-H & 2 & & & 2 \\
\hline & HGSIL & 75 & & & 75 \\
\hline CIN 2 total & & 118 & 41 & & 159 \\
\hline \multirow{7}{*}{ CIN 3} & Inadequate & 8 & 1 & & 9 \\
\hline & Negative & & 2 & & 2 \\
\hline & ASC-US & 2 & 3 & & 5 \\
\hline & LGSIL & 8 & 7 & & 15 \\
\hline & ASC-H & 3 & & & 3 \\
\hline & HGSIL & 94 & & & 94 \\
\hline & SCC & 1 & & & 1 \\
\hline CIN 3 total & & 116 & 13 & & 129 \\
\hline \multirow{5}{*}{ SCC } & Inadequate & 2 & & & 2 \\
\hline & ASC-H & 2 & & & 2 \\
\hline & HGSIL & 9 & & & 9 \\
\hline & SCC & 15 & & & 15 \\
\hline & ADENO-CA & 1 & & & 1 \\
\hline SCC total & & 29 & & & 29 \\
\hline \multirow{6}{*}{ ADENO-CA } & Inadequate & & & & 2 \\
\hline & LGSIL & & 1 & & 1 \\
\hline & ASC-H & & 1 & & 1 \\
\hline & HGSIL & 2 & & & 2 \\
\hline & SCC & 2 & & & 2 \\
\hline & ADENO-CA & 10 & & & 10 \\
\hline ADENO-CA total & & 16 & 2 & & 18 \\
\hline Grand total & & 1495 & 56 & 74 & 1625 \\
\hline
\end{tabular}


of any high risk and of individual subtypes 16 and 62 was found important in our dataset and especially subtype 62 was a discriminating factor for a small number of cases. In relation to E6 and E7 expression, it was found that the flow cytometry results expressed both as positive/negative and as a percentage as well as the subtype 31 from the NASBA examination were important. Moreover, the immunocytochemical expression of p16 and parous data also appeared in the CART branches.

Based on the results, the proposed methodology had superior performance in relation to the overall accuracy $(92.00 \%)$ than the majority of alternative methods (Table 4 ). There was marginal statistically significant difference only between CART and the cytology with ASC-H+ threshold $\left(\chi^{2}=4.027, P=0.0448\right)$. However, for all other comparisons, the differences in the overall accuracy were statistically significant, specifically CART against cytology with ASCUS+ threshold $(P<0.0001)$, arrays using any type $(P<$ $0.0001)$, arrays using high risk subtypes $(P<0.0001)$, arrays for subtype 16 or $18(P<0.0001)$, NASBA for any type $(P<0.0001)$, and finally p16 $(P<0.0001)$. In relation to the comparison of the CART model and the cytological examination with threshold ASC-H+, the sensitivity of the CART model (83.28\%) was significantly higher than cytology (69.33\%); therefore, the proposed method had significantly $(P<0.0001)$ better performance than all other alternatives.

In relation to the false positive cases, the CART wrongly categorized 74 cases as positive ( $\geq$ CIN2); from these 12 were negative and $62 \mathrm{CIN}-1$ in histology, although 50 of these cases were given as ASC-H or HGSIL in cytology (Table 5), 17 as LGSIL, 3 as ASC-US, and the remaining 4 as inadequate in cytology. No case was cytologically negative, as the cytological result is the primary characteristic that is considered as important by our methodology, and these results were expected. The FPR of the CART model was $5.74 \%$, outperformed only by the cytological result with ASCUS+ cutoff (2.24\%) but at the cost of specificity ( 94.26 versus $59.79 \%$; see Table 4).

The analysis of false negative cases is more important; the CART model gave 56 false negative cases in total (14 ASC-US, 32 LGSIL, 2 inadequate, 7 negative in cytology, and 1 ASC$\mathrm{H}$; see Table 5). None of these cases had HGSIL or cancer as cytological result. The histological outcome of these cases was $41 \mathrm{CIN}-2,13 \mathrm{CIN}-3$, and 2 adenocarcinomas. The cytological result for the last 2 cases was ASCH and LGSIL and there was additionally colposcopical agreement.

In relation to the 61 samples that were inadequate in cytology (Table 5), 22 were $\geq$ CIN2, among them 4 carcinomas ( 2 adenocarcinomas and 2 SCC), 9 CIN-2, and 9 CIN-3, and the remaining 39 cases were $<\mathrm{CIN} 2$; the CART model classified correctly 54 of them $(35<\mathrm{CIN} 2,8 \mathrm{CIN}-2,8 \mathrm{CIN}-3,2$ SCC, and 2 adenocarcinomas) and missed 6 cases (4 CIN-1, 1 $\mathrm{CIN}-2$, and $1 \mathrm{CIN}-3$ ). It is worth noting that this decision was based only on biomarker data and parous status. Therefore, the number of women that would require a second cytological examination could be reduced dramatically.

Concluding the application of the proposed method gave encouraging results and not only could be helpful towards a better management of women for various findings during cytological examination but also provides a flexible technique for the estimation of $\geq \mathrm{CIN} 2$ risk. As a result, the proposed method provides a guide towards personalized management and therapeutic decisions, may reduce the overload of colposcopy clinics and unnecessary treatments, and identifies a higher percentage of women at risk of cervical cancer or precancerous lesions.

\section{Conflict of Interests}

All authors declare that there is no conflict of interests.

\section{Acknowledgments}

This study was partially funded by the Greek Ministry of Development (General Secretariat for Research and Technology (GSRT)), Project acronym: HPV-Guard (Cooperation

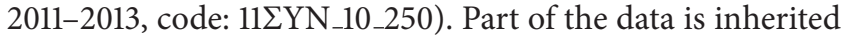
from two other funded research projects: Project "AKAKOS" (code: ATT 95) funded by GSRT and "Study and Evaluation of the Methods, for the Prognosis of Success for Vaccination against HPV Infections-Ygeia Proneia, 2000-2006" funded from the Greek Ministry of Health. Additional data is obtained from the HeCPA (Hellenic Cervical Pathology Academic) Study Group. MK is supported by the Imperial Healthcare NHS Biomedical Research Grant (P45272), the Imperial College Healthcare Charity Fellowship and received research awards from the British Society of Colposcopy and Cervical Pathology.

\section{References}

[1] A. Jemal, F. Bray, M. M. Center, J. Ferlay, E. Ward, and D. Forman, "Global cancer statistics," CA: Cancer Journal for Clinicians, vol. 61, no. 2, pp. 69-90, 2011.

[2] W. A. Leyden, M. M. Manos, A. M. Geiger et al., "Cervical cancer in women with comprehensive health care access: attributable factors in the screening process," Journal of the National Cancer Institute, vol. 97, no. 9, pp. 675-683, 2005.

[3] H. L. Smits, B. van Gemen, R. Schukkink et al., "Application of the NASBA nucleic acid amplification method for the detection of human papillomavirus type 16 E6-E7 transcripts," Journal of Virological Methods, vol. 54, no. 1, pp. 75-81, 1995.

[4] A. Tropé, K. Sjøborg, A. Eskild et al., "Performance of human papillomavirus DNA and mRNA testing strategies for women with and without cervical neoplasia," Journal of Clinical Microbiology, vol. 47, no. 8, pp. 2458-2464, 2009.

[5] S. W. Sørbye, S. Fismen, T. Gutteberg, and E. S. Mortensen, "Triage of women with minor cervical lesions: data suggesting a 'test and treat' approach for HPV E6/E7 mRNA testing," PLoS ONE, vol. 5, no. 9, Article ID e12724, pp. 1-6, 2010.

[6] R. Narimatsu and B. K. Patterson, "High-throughput cervical cancer screening using intracellular human papillomavirus e6 and e7 mRNA quantification by flow cytometry," The American Journal of Clinical Pathology, vol. 123, no. 5, pp. 716-723, 2005.

[7] G. Coquillard, B. Palao, and B. K. Patterson, "Quantification of intracellular HPV E6/E7 mRNA expression increases the specificity and positive predictive value of cervical cancer screening compared to HPV DNA," Gynecologic Oncology, vol. 120, no. 1, pp. 89-93, 2011. 
[8] S. Nieh, S. F. Chen, T. Y. Chu et al., "Is p16INK4A expression more useful than human papillomavirus test to determine the outcome of atypical squamous cells of undetermined significance-categorized Pap smear? A comparative analysis using abnormal cervical smears with follow-up biopsies," Gynecologic Oncology, vol. 97, no. 1, pp. 35-40, 2005.

[9] M.-H. Mayrand, E. Duarte-Franco, I. Rodrigues et al., "Human papillomavirus DNA versus Papanicolaou screening tests for cervical cancer," The New England Journal of Medicine, vol. 357, no. 16, pp. 1579-1588, 2007.

[10] A. Mathew and P. S. George, "Trends in incidence and mortality rates of squamous cell carcinoma and adenocarcinoma of cervix-worldwide," Asian Pacific Journal of Cancer Prevention, vol. 10, no. 4, pp. 645-650, 2009.

[11] J. Cuzick, M. Arbyn, R. Sankaranarayanan et al., "Overview of human papillomavirus-based and other novel options for cervical cancer screening in developed and developing countries," Vaccine, vol. 26, no. 10, pp. K29-K41, 2008.

[12] P. Naucler, W. Ryd, S. Törnberg et al., "Efficacy of HPV DNA testing with cytology triage and/or repeat hpv DNA testing in primary cervical cancer screening," Journal of the National Cancer Institute, vol. 101, no. 2, pp. 88-99, 2009.

[13] M. Benevolo, A. Vocaturo, D. Caraceni et al., "Sensitivity, specificity, and clinical value of human papillomavirus (HPV) E6/E7 mRNA assay as a triage test for cervical cytology and HPV DNA test," Journal of Clinical Microbiology, vol. 49, no. 7, pp. 2643-2650, 2011.

[14] K. Cuschieri and N. Wentzensen, "Human papillomavirus mRNA and p16 detection as biomarkers for the improved diagnosis of cervical neoplasia," Cancer Epidemiology Biomarkers and Prevention, vol. 17, no. 10, pp. 2536-2545, 2008.

[15] I. Tsoumpou, M. Arbyn, M. Kyrgiou et al., "p16INK4a immunostaining in cytological and histological specimens from the uterine cervix: a systematic review and meta-analysis," Cancer Treatment Reviews, vol. 35, no. 3, pp. 210-220, 2009.

[16] F. M. Carozzi, "Combined analysis of HPV DNA and p16INK4a expression to predict prognosis in ASCUS and LSIL pap smears," Collegium Antropologicum, vol. 31, supplement 2, pp. 103-106, 2007.

[17] K. J. Denton, C. Bergeron, P. Klement, M. J. Trunk, T. Keller, and R. Ridder, "The sensitivity and specificity of $16^{I N K 4 a}$ cytology vs HPV testing for detecting high-grade cervical disease in the triage of asc-us and lsil pap cytology results," The American Journal of Clinical Pathology, vol. 134, no. 1, pp. 12-21, 2010.

[18] A. M. Marchevsky, J. A. Tsou, and I. A. Laird-Offringa, "Classification of individual lung cancer cell lines based on DNA methylation markers: use of linear discriminant analysis and artificial neural networks," Journal of Molecular Diagnostics, vol. 6, no. 1, pp. 28-36, 2004.

[19] A. Errejon, E. D. Crawford, J. Dayhoff et al., "Use of artificial neural networks in prostate cancer," Molecular Urology, vol. 5, no. 4, pp. 153-158, 2001.

[20] C. S. Gong, L. Yu, C. K. Ting et al., "Predicting postoperative vomiting for orthopedic patients receiving patient-controlled epidural analgesia with the application of an artificial neural network," BioMed Research International, vol. 2014, Article ID 786418, 6 pages, 2014.

[21] D. I. Gheonea, C. T. Streba, C. C. Vere et al., "Diagnosis system for hepatocellular carcinoma based on fractal dimension of morphometric elements integrated in an artificial neural network," BioMed Research International, vol. 2014, Article ID 239706, 10 pages, 2014.
[22] K. P. Ferentinos, C. P. Yialouris, P. Blouchos, G. Moschopoulou, and S. Kintzios, "Pesticide residue screening using a novel artificial neural network combined with a bioelectric cellular biosensor," BioMed Research International, vol. 2013, Article ID 813519, 8 pages, 2013.

[23] L. Zhu, W. Luo, M. Su et al., "Comparison between artificial neural network and Cox regression model in predicting the survival rate of gastric cancer patients," Biomedical Reports, vol. 1, pp. 757-760, 2013.

[24] Z. Amiri, K. Mohammad, M. Mahmoudi, H. Zeraati, and A. Fotouhi, "Assessment of gastric cancer survival: using an artificial hierarchical neural network," Pakistan Journal of Biological Sciences, vol. 11, no. 8, pp. 1076-1084, 2008.

[25] E. H. Bollschweiler, S. P. Mönig, K. Hensler, S. E. Baldus, K. Maruyama, and A. H. Hölscher, "Artificial neural network for prediction of lymph node metastases in gastric cancer: a phase II diagnostic study," Annals of Surgical Oncology, vol. 11, no. 5, pp. 506-511, 2004.

[26] P. Karakitsos, A. Kyroudes, A. Pouliakis, E. Botsoli Stergiou, Z. Voulgaris, and C. Kittas, "Potential of the learning vector quantizer in the cell classification of endometrial lesions in postmenopausal women," Analytical and Quantitative Cytology and Histology, vol. 24, no. 1, pp. 30-38, 2002.

[27] L.-C. Zhu, Y.-L. Ye, W.-H. Luo et al., "A model to discriminate malignant from benign thyroid nodules using artificial neural network," PLoS ONE, vol. 8, no. 12, Article ID e82211, 2013.

[28] R. M. Subbaiah, P. Dey, and R. Nijhawan, "Artificial neural network in breast lesions from fine-needle aspiration cytology smear," Diagnostic Cytopathology, vol. 42, no. 3, pp. 218-224, 2014.

[29] P. Dey, R. Logasundaram, and K. Joshi, "Artificial neural network in diagnosis of lobular carcinoma of breast in fine-needle aspiration cytology," Diagnostic Cytopathology, vol. 41, no. 2, pp. 102-106, 2013.

[30] E. S. McHugh, A. P. Shinn, and J. W. Kay, "Discrimination of the notifiable pathogen Gyrodactylus salaris from G. thymalli (Monogenea) using statistical classifiers applied to morphometric data," Parasitology, vol. 121, no. 3, pp. 315-323, 2000.

[31] S. Datta, "Classification of breast cancer versus normal samples from mass spectrometry profiles using linear discriminant analysis of important features selected by random forest," Statistical Applications in Genetics and Molecular Biology, vol. 7, no. 2, 2008.

[32] J. M. le Goff, L. Lavayssière, J. Rouëssé, and F. Spyratos, "Nonlinear discriminant analysis and prognostic factor classification in node-negative primary breast cancer using probabilistic neural networks," Anticancer Research, vol. 20, no. 3, pp. 2213-2218, 2000.

[33] J. P. Marques de Sá, Applied Statistics: Using SPSS, STATISTICA, MATLAB, and R, Springer, Berlin, Germany, 2nd edition, 2007.

[34] L. Breiman, J. Friedman, C. J. Stone, and R. A. Olshen, Classification and regression trees, Wadsworth International Group, Belmont, Calif, USA, 1984.

[35] P. Karakitsos, A. Pouliakis, C. Meristoudis et al., "A preliminary study of the potential of tree classifiers in triage of high-grade squamous intraepithelial lesions," Analytical and Quantitative Cytology and Histology, vol. 33, no. 3, pp. 132-140, 2011.

[36] F. Saiti, A. A. Naini, M. A. Shoorehdeli, and M. Teshnehlab, "Thyroid disease diagnosis based on genetic algorithms using PNN and SVM," in Proceedings of the 3rd International Conference on Bioinformatics and Biomedical Engineering (ICBBE '09), June 2009. 
[37] F. C. Schmitt, A. Longatto-Filho, A. Valent, and P. Vielh, "Molecular techniques in cytopathology practice," Journal of Clinical Pathology, vol. 61, no. 3, pp. 258-267, 2008.

[38] M. R. Henry, "The bethesda system 2001: an update of new terminology for gynecologic cytology," Clinics in Laboratory Medicine, vol. 23, no. 3, pp. 585-603, 2003.

[39] J. H. F. Smith, “Bethesda 2001," Cytopathology, vol. 13, no. 1, pp. 4-10, 2002.

[40] J. J. Gomez-Roman, C. Echevarria, S. Salas et al., "A type-specific study of human papillomavirus prevalence in cervicovaginal samples in three different Spanish regions," APMIS, vol. 117, no. 1, pp. 22-27, 2009.

[41] S. Tyagi, D. P. Bratu, and F. R. Kramer, "Multicolor molecular beacons for allele discrimination," Nature Biotechnology, vol. 16, no. 1, pp. 49-53, 1998.

[42] R. Klaes, A. Benner, T. Friedrich et al., "p16INK4a immunohistochemistry improves interobserver agreement in the diagnosis of cervical intraepithelial neoplasia," The American Journal of Surgical Pathology, vol. 26, no. 11, pp. 1389-1399, 2002.

[43] H. F. Nauth, Gynecological Cytology, Georg Thieme, Stuttgart, Germany, 2007.

[44] M. Arbyn, P. Martin-Hirsch, F. Buntinx, M. van Ranst, E. Paraskevaidis, and J. Dillner, "Triage of women with equivocal or low-grade cervical cytology results: a meta-analysis of the HPV test positivity rate," Journal of Cellular and Molecular Medicine, vol. 13, no. 4, pp. 648-659, 2009.

[45] M. Arbyn, F. Buntinx, M. van Ranst, E. Paraskevaidis, P. MartinHirsch, and J. Dillner, "Virologic versus cytologic triage of women with equivocal pap smears: a meta-analysis of the accuracy to detect high-grade intraepithelial neoplasia," Journal of the National Cancer Institute, vol. 96, no. 4, pp. 280-293, 2004.

[46] J. Roelens, M. Reuschenbach, M. von Knebel Doeberitz, N. Wentzensen, C. Bergeron, and M. Arbyn, "P16INK4a immunocytochemistry versus human papillomavirus testing for triage of women with minor cytologic abnormalities: a systematic review and meta-analysis," Cancer Cytopathology, vol. 120, no. 5, pp. 294-307, 2012.

[47] J. L. Brandsma, M. Harigopal, N. B. Kiviat et al., "Methylation of twelve CpGs in human papillomavirus type 16 (HPV16) as an informative biomarker for the triage of women positive for HPV16 infection," Cancer Prevention Research, vol. 7, no. 5, pp. 526-533, 2014.

[48] S. Loghavi, A. E. Walts, and S. Bose, "CINtec PLUS dual immunostain: a triage tool for cervical pap smears with atypical squamous cells of undetermined significance and low grade squamous intraepithelial lesion," Diagnostic Cytopathology, vol. 41, no. 7, pp. 582-587, 2013.

[49] K. A. Ault, H. K. Allen, S. L. Phillips, M. B. Zimmerman, and A. J. Klingelhutz, "Telomerase activity as a potential diagnostic marker for triage of abnormal pap smears," Journal of Lower Genital Tract Disease, vol. 9, no. 2, pp. 93-99, 2005.

[50] H. Lin, T. C. Chen, T. C. Chang et al., "Methylated znf582 gene as a marker for triage of women with pap smear reporting lowgrade squamous intraepithelial lesions - a taiwanese gynecologic oncology group (TGOG) study," Gynecologic Oncology, vol. 135, no. 1, pp. 64-68, 2014.

[51] J. L. Killeen, T. Dye, C. Grace, and M. Hiraoka, "Improved abnormal pap smear triage using cervical cancer biomarkers," Journal of Lower Genital Tract Disease, vol. 18, no. 1, pp. 1-7, 2014.

[52] M. Arbyn, M. Kyrgiou, C. Simoens et al., "Perinatal mortality and other severe adverse pregnancy outcomes associated with treatment of cervical intraepithelial neoplasia: meta-analysis," British Medical Journal, vol. 337, article a1284, 2008.

[53] R. S. Hoda and S. A. Hoda, Fundamentals of Pap Test Cytology, Humana Press, Totowa, NJ, USA, 2007.

[54] London Quality Assurance Reference Centre, Kc61 Report London Annual Report 2005-2006, London Quality Assurance Reference Centre, London, UK, 2006.

[55] B. A. Jones and D. A. Novis, "Cervical biopsy-cytology correlation: a College of American Pathologists Q-Probes study of 22439 correlations in 348 laboratories," Archives of Pathology \& Laboratory Medicine, vol. 120, no. 6, pp. 523-531, 1996.

[56] P. Karakitsos, C. Chrelias, A. Pouliakis et al., "Identification of women for referral to colposcopy by neural networks: a preliminary study based on LBC and molecular biomarkers," Journal of Biomedicine \& Biotechnology, vol. 2012, Article ID 303192, 8 pages, 2012.

[57] P. Bountris, M. Haritou, A. Pouliakis et al., "An intelligent clinical decision support system for patient-specific predictions to improve cervical intraepithelial neoplasia detection," BioMed Research International, vol. 2014, Article ID 341483, 20 pages, 2014. 


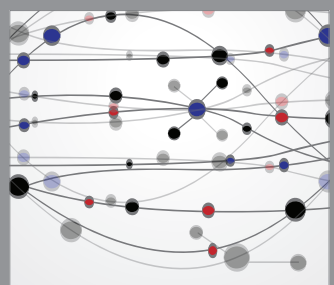

The Scientific World Journal
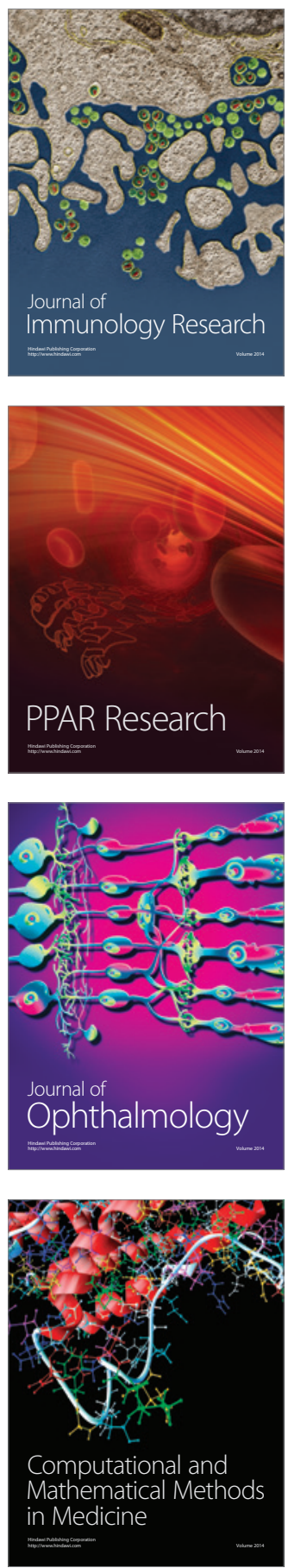

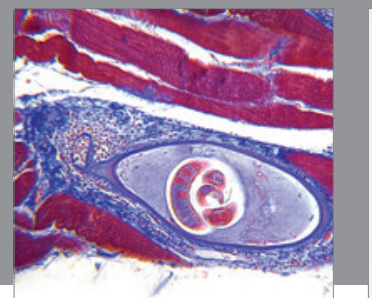

Gastroenterology

Research and Practice
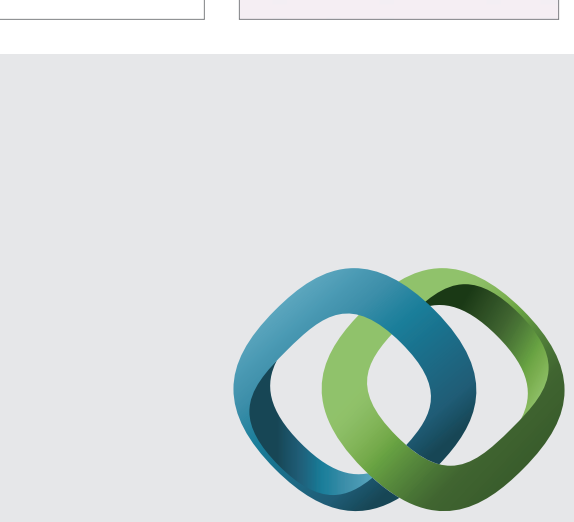

\section{Hindawi}

Submit your manuscripts at

http://www.hindawi.com
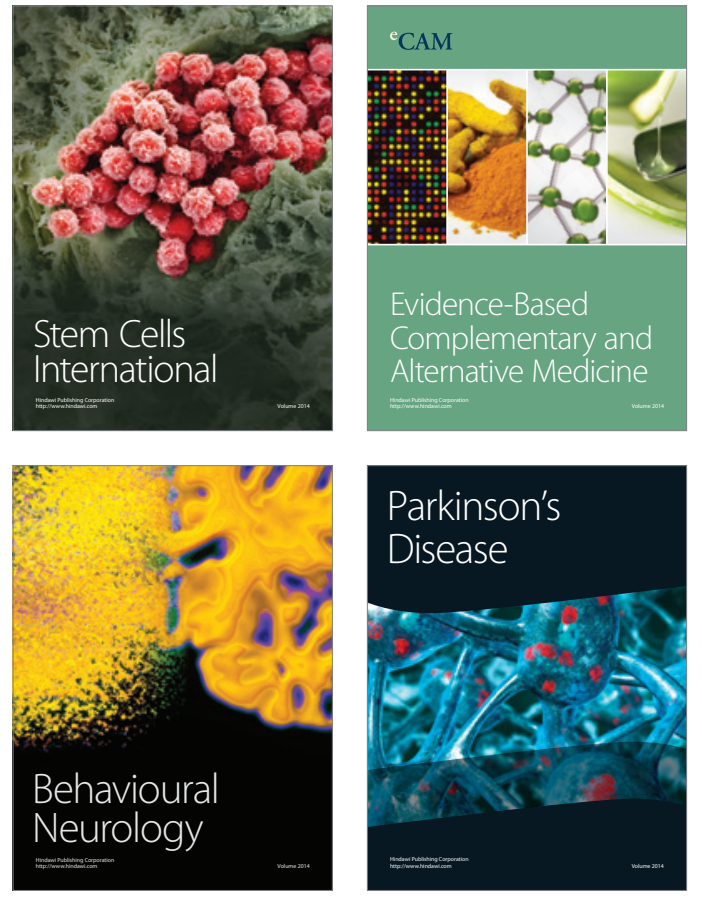
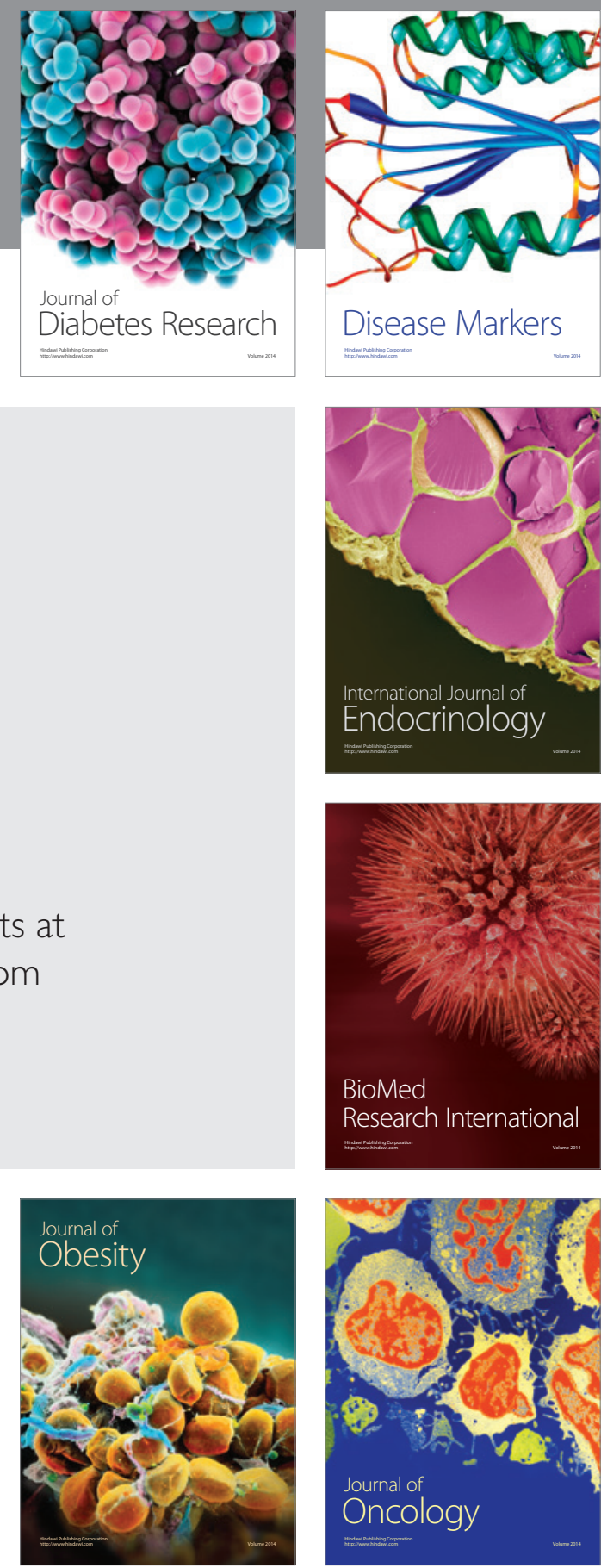

Disease Markers
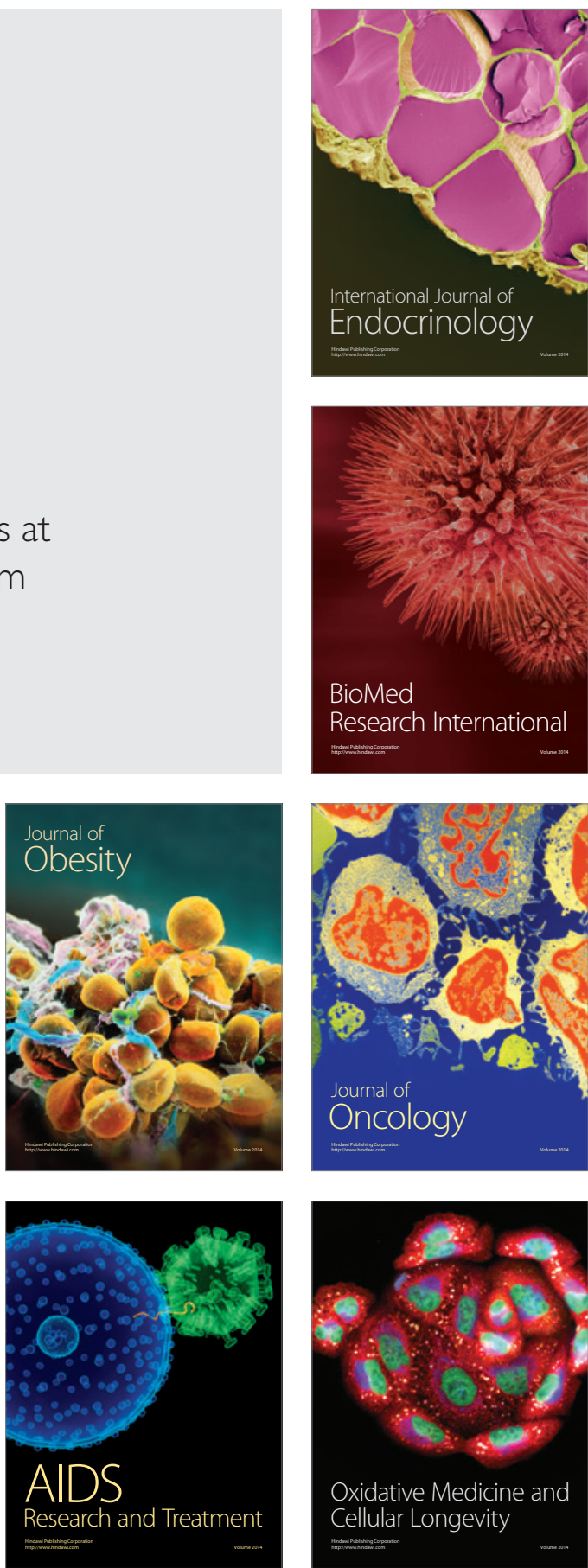